\title{
Glucose metabolism controls disease-specific signatures of macrophage effector functions
}

Ryu Watanabe, ${ }^{1}$ Marc Hilhorst,, ${ }^{1,2}$ Hui Zhang, ${ }^{1}$ Markus Zeisbrich, ${ }^{1}$ Cerald J. Berry, ${ }^{3}$ Barbara B. Wallis, ${ }^{1}$ David C. Harrison, ${ }^{4}$ John C. Giacomini, ${ }^{5}$ Jörg J. Goronzy, ${ }^{1}$ and Cornelia M. Weyand ${ }^{1}$

'Department of Medicine, Division of Immunology and Rheumatology, Stanford University School of Medicine, Stanford, California, USA. ${ }^{2}$ Department of Internal Medicine, Academisch Medisch Centrum Universiteit van Amsterdam, Amsterdam, Netherlands. ${ }^{3}$ Department of Pathology, Stanford University School of Medicine, Stanford, California, USA. ${ }^{4}$ Division of Clinical Pharmacology, Department of Medicine, Vanderbilt University School of Medicine, Nashville, Tennessee, USA. ${ }^{5}$ Division of Cardiovascular Medicine, Department of Medicine, Stanford University School of Medicine, Stanford, California USA.

BACKGROUND. In inflammatory blood vessel diseases, macrophages represent a key component of the vascular infiltrates and are responsible for tissue injury and wall remodeling.

METHODS. To examine whether inflammatory macrophages in the vessel wall display a single distinctive effector program, we compared functional profiles in patients with either coronary artery disease (CAD) or giant cell arteritis (CCA).

RESULTS. Unexpectedly, monocyte-derived macrophages from the 2 patient cohorts displayed disease-specific signatures and differed fundamentally in metabolic fitness. Macrophages from CAD patients were high producers for T cell chemoattractants (CXCL9, CXCL10), the cytokines IL-1 $\beta$ and IL-6, and the immunoinhibitory ligand PD-L1. In contrast, macrophages from CCA patients upregulated production of T cell chemoattractants (CXCL9, CXCL10) but not IL-1 $\beta$ and IL-6, and were distinctly low for PD-L1 expression. Notably, disease-specific effector profiles were already identifiable in circulating monocytes. The chemokine ${ }^{\text {hi }}{ }^{2}$ tokine ${ }^{\text {hiPD- }}$ L1 $^{\text {hi }}$ signature in CAD macrophages was sustained by excess uptake and breakdown of glucose, placing metabolic control upstream of inflammatory function.

CONCLUSIONS. We conclude that monocytes and macrophages contribute to vascular inflammation in a disease-specific and discernible pattern, have choices to commit to different functional trajectories, are dependent on glucose availability in their immediate microenvironment, and possess memory in their lineage commitment.

FUNDING. Supported by the NIH (R01 AR042527, R01 HL117913, R01 Al108906, P01 HL129941, R01 Al108891, R01 AG045779 U19 AI057266, R01 Al129191), I01 BX001669, and the Cahill Discovery Fund.

Conflict of interest: The authors have declared that no conflict of interest exists.

License: Copyright 2018, American Society for Clinical Investigation.

Submitted: June 20, 2018 Accepted: September 11, 2018 Published: October 18, 2018

\section{Reference information:} JCI Insight. 2018;3(20):e123047. https://doi.org/10.1172/jici. insight.123047.

\section{Introduction}

Macrophages derive from monocytic precursors in the bone marrow and blood and play a key role in both innate and adaptive immunity $(1,2)$. Following infiltration into tissue, monocytes can differentiate into macrophages, acquire phagocytic capacity, and serve as antigen-presenting cells. In addition, tissue macrophages release a range of mediators that have cytotoxic, proinflammatory, and antiinflammatory functions, and promote tissue-digestive, angiogenic, and fibrogenic pathways (2). Macrophage populations are now classified into 2 major subtypes: classically activated M1 and alternatively activated M2 macrophages (3). These 2 states can be induced by stimulation with IFN- $\gamma$ and Toll-like receptor (TLR) ligands for M1, or with IL-4 and IL-13 for M2 (3). M1 macrophages increase and sustain inflammatory responses via the secretion of proinflammatory cytokines such as IL-1 $\beta$, IL- 6 , TNF- $\alpha$, as well as IL-12 and IL-23 (4). M1 macrophages also produce toxic agents such as nitric oxide and ROS to eradicate 
bacteria, viruses, and fungi. An important element of inducing inflammatory lesions in tissue is the production of chemokines such as C-X-C motif ligand 9 (CXCL9) and CXCL10 that coordinate the recruitment of Th1 cells, typically amplifying tissue inflammation and impairing wound healing (5). In contrast, M2 macrophages are believed to counteract the inflammatory response induced by M1 macrophages and instead facilitate wound healing and tissue repair through the high production of IL-10 and their proangiogenic and profibrotic capacities (6).

Giant cell arteritis (GCA) is the prototypic large-vessel vasculitis that manifests in the aorta and the major branches $(7,8)$. The typical histopathology of affected arteries includes transmural infiltrates composed of $\mathrm{CD}^{+} \mathrm{T}$ cells and highly activated macrophages, often arranged in granulomatous lesions with multinucleated giant cells. Minor cell populations include mast cells, NK cells, and B cells. High proliferation rates of lesional $\mathrm{T}$ cells suggest that vasculitogenic T cells are locally stimulated (9). Antigen-presenting function has been assigned to an endogenous population of dendritic cells, so-called vascular dendritic cells (10), and identical $\mathrm{T}$ cell receptor sequences expressed by $\mathrm{T}$ cells in distinct vasculitic sites have supported the concept of disease-relevant antigens driving the vasculitic process (11). T effector cells recruited to the vessel wall typically produce a variety of inflammatory cytokines, including IFN- $\gamma$, IL-17, IL-21, and IL-9 (12-14), which has given rise to the hypothesis that threshold setting in the adaptive immune system is defective due to a loss of immune checkpoint function (15). In line with a broad spectrum of effector $\mathrm{T}$ cells, the vasculitic lesions contain functionally diverse macrophage populations. Highly activated macrophages and their fusion product, multinucleated giant cells, secrete cytokines, chemokines, metalloproteinases, angiogenic factors, growth factors, ROS, and aldose reductase (16-18). Gene expression analysis of vasculitic arteries has demonstrated the dominance of gene products induced by type 1 and type 2 IFNs, including the chemokines CXCL9 and CXCL10 $(9,16)$. Inflammation-induced wall remodeling leads to luminal stenosis/occlusion through intimal hyperplasia, a process accompanied by digestion of the elastic laminae and by vigorous neoangiogenesis.

Like large-vessel vasculitis, atherosclerosis is a fibroinflammatory vasculopathy that affects the aorta and such vascular beds as the epicardial coronary, carotid, intracerebral, and femoral arteries (19). Macrophages have disease-relevant functions at all stages of the disease process, beginning with the uptake of subendothelially deposited lipids (20). Lesional macrophages phagocytose low-density lipoproteins and form lipid-laden foam cells (21). In general, M1 macrophages promote atherosclerosis progression, whereas M2 macrophages suppress plaque progression and promote plaque regression (22). M1 macrophages produce proinflammatory cytokines, chemokines, proteinases, and ROS and contribute to tissue damage manifesting as plaque instability (23). Recent molecular profiling studies have emphasized the heterogeneity and plasticity of macrophages in the atherosclerotic plaque (21).

Given the similarities in the arterial wall tissue microenvironment, and the similarities in wall remodeling, the question arises as to whether the pathogenic contribution of macrophages in vasculitis and atherosclerosis is similar. The activation program of macrophages involves upregulation of the metabolic machinery to provide the energy and the biosynthetic precursors required for the production of effector molecules, such as cytokines, chemokines, digestive enzymes, etc. (20). Macrophages from patients with coronary artery disease (CAD) respond to activation with the induction of a gene module that includes the glucose transporter GLUT1, a series of glycolytic enzymes, and the transcriptional regulators c-myc and hypoxia-inducible factor $1 \alpha(\mathrm{HIF}-1 \alpha)$ (20). Commitment to high glucose utilization has functional consequences; e.g., mitochondrial ROS oxidize cytoplasmic pyruvate kinase M2, to enable nuclear translocation and STAT3 activation and the secretion of IL-1 $\beta$ and IL-6 (24). Access to high extracellular glucose concentrations also drives expression of the immunoinhibitory ligand PD-L1, such that macrophages from $\mathrm{CAD}$ patients suppress antiviral $\mathrm{T}$ cell responses (25).

By comparing monocytes and monocyte-derived macrophages from patients with either CAD or GCA, we defined a disease-specific effector panel for each condition. Macrophages from CAD patients excelled in producing chemokines and cytokines, in particular IL-6, but also expressed high amounts of PD-L1, thus combining the ability to send pro- and antiinflammatory signals. As a distinguishing feature, they were highly efficient in the uptake and breakdown of glucose, which directly fueled cytokine release and PD-L1 expression. In contrast, macrophages from GCA patients resembled those from healthy controls in glucose uptake and glycolytic activity and were distinctly low in PD-L1 expression, thus failing in the termination of immune responses. Prone to producing chemokines, GCA macrophages produced cytokines similar to healthy controls and may primarily aid in the building of inflammatory infiltrates. The commitment to 

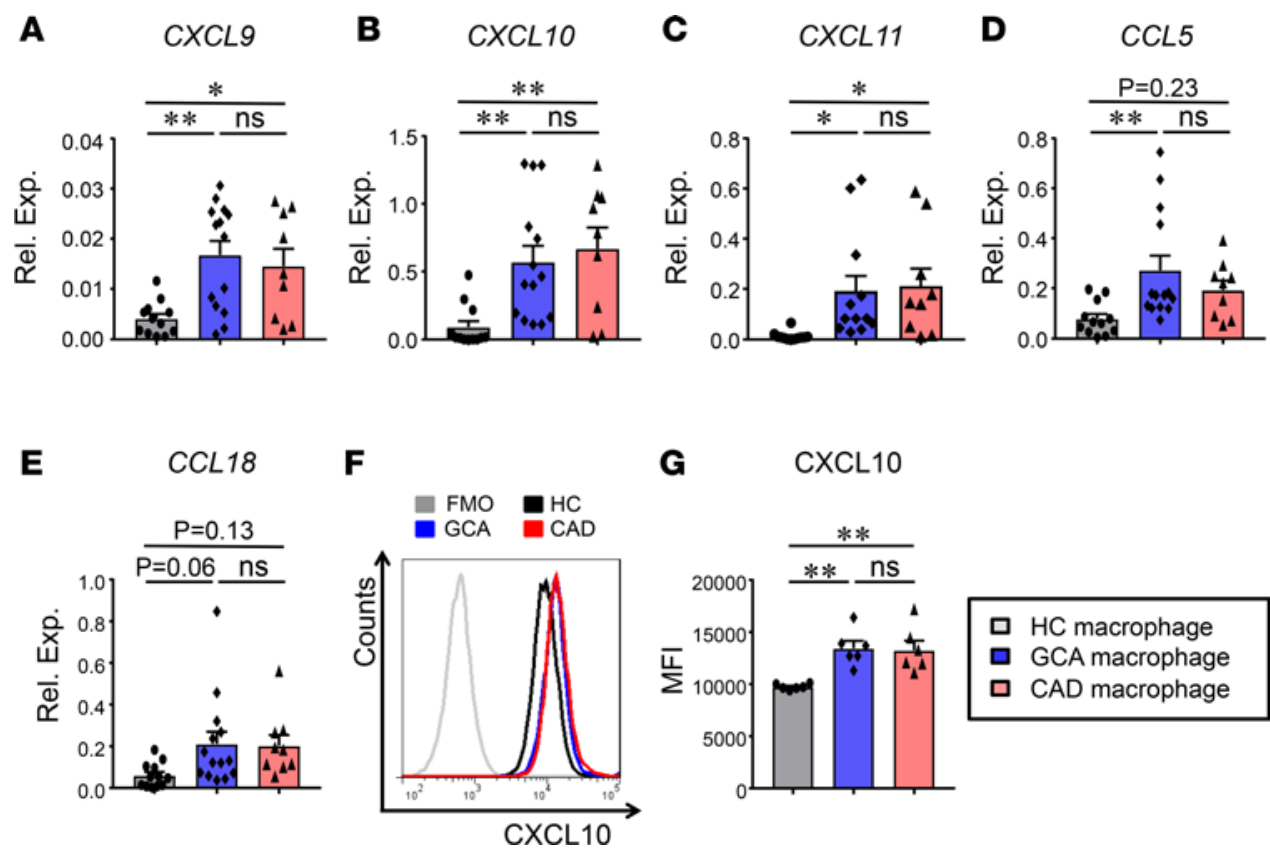

HC macrophage
$\square$ GCA macrophage
$\square$ CAD macrophage

Figure 1. Similar patterns of chemokine production in monocyte-derived macrophages from patients with GCA or CAD. Macrophages were differentiated ex vivo from peripheral blood monocytes of patients with GCA, patients with CAD, and age-matched healthy controls and were stimulated with LPS/IFN- $\gamma$ for 24 hours. (A-E) Gene transcripts for CXCL9, CXCL10, CXCL11, CCL5, and CCL18 were measured by RT-PCR. Data are from 12 HC, 14 GCA, and 9 (including 5 diabetic) CAD samples. (F and $\mathbf{G}$ ) Intracellular CXCL10 protein concentrations measured by flow cytometry. (F) Representative histograms. (C) Summary results from 6 (including 3 diabetic) CAD patients, 6 GCA patients, and 6 age-matched controls. All data are mean \pm SEM and were analyzed by 1-way ANOVA with Tukey's multiple comparison test. ${ }^{*} P<$ $0.05,{ }^{* *} P<0.01$. CAD, coronary artery disease; CCL, C-C motif chemokine ligand; CXCL, C-X-C motif chemokine ligand; FMO, fluorescence minus one; GCA, giant cell arteritis; HC, healthy control; IFN- $\gamma$, interferon $\gamma$; LPS, lipopolysaccharide; MFI, mean fluorescence intensity; ns, not significant; Rel. Exp., relative expression; RT-PCR, reverse transcription polymerase chain reaction.

glycolysis and the pattern of chemokine and cytokine production was sustained from monocytic precursors to tissue-resident macrophages, compatible with imprinting of the metabolic program as a mechanism of memory building in monocytes and macrophages.

\section{Results}

GCA and CAD macrophages are prone to producing chemokines. The vessel wall lesions in GCA and in CAD typically contain chemokine-producing macrophages that sustain recruitment to build $\mathrm{T}$ cell-rich chronic inflammatory lesions $(16,26)$. We generated macrophages from peripheral blood CD14+ monocytes of 3 study cohorts - patients with CAD, patients with GCA, and age-matched healthy controls - and activated them with IFN- $\gamma /$ LPS for 24 hours. Transcriptome analysis showed that patient-derived macrophages from both CAD and GCA patients produced higher amounts of the chemokines CXCL9, CXCL10, and CXCL11, which facilitate the recruitment of Th1 cells (Figure 1, A-C). Compared with macrophages from healthy individuals, patient-derived macrophages from both groups tended to be more efficient in the transcription of chemokine (C-C motif) ligand 5 (CCL5) and CCL18 (Figure 1, D and E). Flow cytometric analysis confirmed that patient-derived macrophages had significantly higher concentrations of intracellular CXCL10 protein in both patient groups (Figure 1, F and G).

To understand whether the preference for CXC chemokine production is maintained in macrophages that have entered the tissue site in the blood vessel wall, we examined tissue sections from GCA-affected temporal arteries and from carotid atheromas (Figure 2). Analysis of gene transcripts for the CXC family members CXCL9, CXCL10, and CXCL11 revealed that noninflamed human medium-sized arteries were essentially negative for CXCL10-specific sequences; CXCL9 was transcribed at low abundance, whereas CXCL11 transcripts were highly abundant (Figure 2, A-C). All 3 family members were present at high abundance in vasculitic arteries. Similarly, carotid atheromas were rich in CXCL9, CXCL10, and CXCL11 

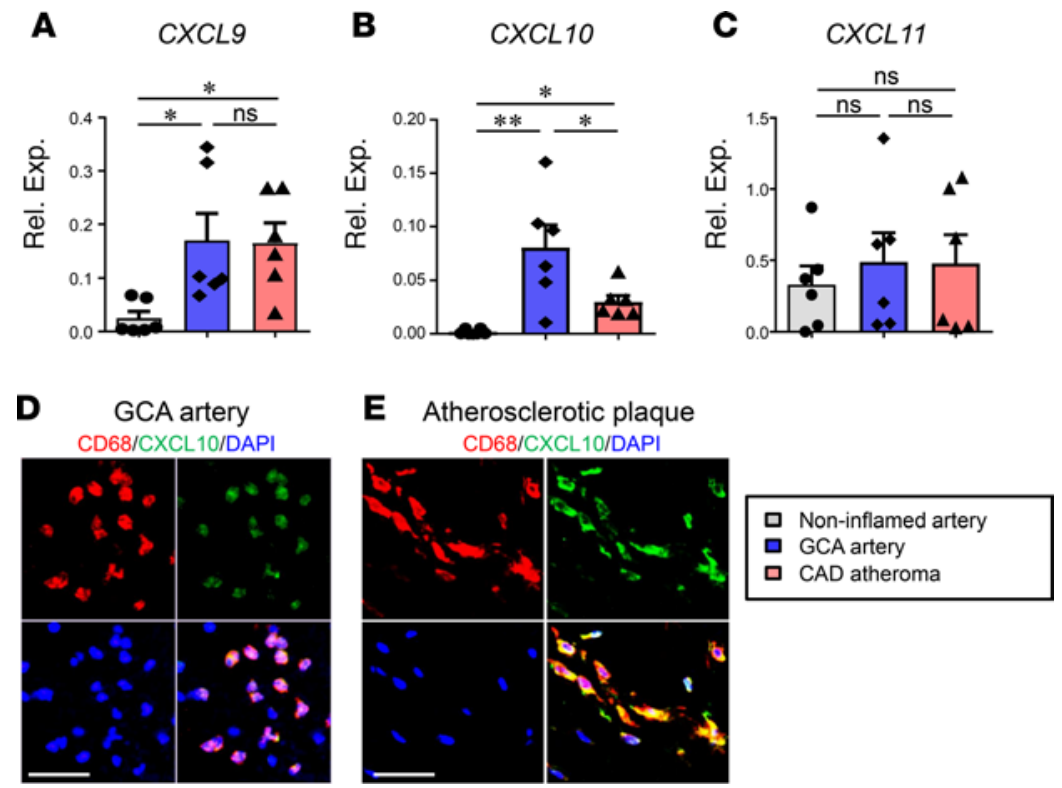

Figure 2. Chemokine production in vasculitic wall lesions and in atherosclerotic plaques. (A-C) Transcripts specific for CXCL9, CXCL10, and CXCL11 were quantified by RT-PCR in tissue extracts from noninflamed human arteries, GCA-affected temporal arteries, and carotid atheromas ( $n=6$ each). Data are mean \pm SEM. (D and E) Dual immunostaining of CD68 (red) and CXCL10 (green) in tissue sections from GCA-affected temporal arteries (D) and aortic atherosclerotic plaques (E). Nuclei were labeled with DAPI. Scale bars: $50 \mu \mathrm{m}$. Data were analyzed by 1-way ANOVA with Tukey's multiple comparison test. ${ }^{*} P<0.05,{ }^{* *} P<0.01$. CAD, coronary artery disease; CXCL, C-X-C motif chemokine ligand; CCA, giant cell arteritis; ns, not significant; Rel. Exp., relative expression; RT-PCR, reverse transcription polymerase chain reaction.

transcripts. Notably, CXCL10 transcripts were particularly prevalent in inflamed temporal arteries compared with atheromatous lesions. Immunohistochemical staining demonstrated that in both disease states CD68 ${ }^{+}$cells were strongly positive for CXCL10 (Figure 2, D and E). Production of CXCL10 protein was a feature of the majority of $\mathrm{CD} 68^{+}$cells, most of which are macrophages.

In summary, macrophages from patients with GCA and CAD share the ability to produce $\mathrm{T}$ cell chemoattractants, enabling them to promote the enrichment of adaptive immune cells in the tissue niche of the vessel wall.

CAD macrophages excel at the production of IL-1 $\beta$ and IL-6. Both GCA and CAD are associated with elevation of the acute phase reactant $\mathrm{C}$-reactive protein (CRP), a biomarker of disease burden that is induced by the cytokine IL-6 $(27,28)$. In CAD patients, plasma CRP levels correlate with IL-6 released by activated macrophages (24). Disease relevance of the cytokine IL-1 $\beta$ is supported by the results of a recent randomized trial that demonstrated that canakinumab, a monoclonal antibody targeting IL-1 $\beta$, prevented recurrence of cardiovascular events (29). To examine whether macrophages are a potential cellular source of the proinflammatory cytokines IL-1 $\beta$, IL- 6 , and TNF- $\alpha$, we generated monocyte-derived macrophages from GCA and CAD patients and from age-matched healthy controls, activated them with IFN- $\gamma / \mathrm{LPS}$, and determined transcript levels for the 3 cytokines (Figure 3). Macrophages derived from GCA patients were indistinguishable from control macrophages (Figure 3, A-C). Conversely, CAD macrophages displayed a pattern of highly abundant transcripts for IL-1 $\beta$ and IL-6. Relative expression of TNF- $\alpha$-specific sequences was similar in both patient-derived groups and control macrophages. Flow cytometric analysis of intracellular IL-6 protein confirmed that CAD macrophages excel in IL-6 production (Figure 3, D and E). These data established a CAD-specific macrophage effector profile that includes IL- $1 \beta$ and IL- 6 , in line with clinical data implicating these cytokines in the pathogenesis of atherosclerotic disease. Unexpectedly, macrophages appear not to be the dominant producer cells for IL-1 $\beta$ and IL-6 in patients with vasculitis.

CAD and GCA macrophages are fundamentally different in their expression of the immunoinhibitory ligand PD-L1. Besides secreting chemokines and cytokines, macrophages regulate neighboring cells by expressing cell surface receptors/ligands. Specifically, through CD80 and CD86, macrophages enhance T cell activation, and provide negative signals by expressing the inhibitory ligand PD-L1 (30). PD-L1 expression on CAD macrophages has been associated with inhibition of antiviral $\mathrm{T}$ cell responses (25). We generated monocyte-derived macrophages 

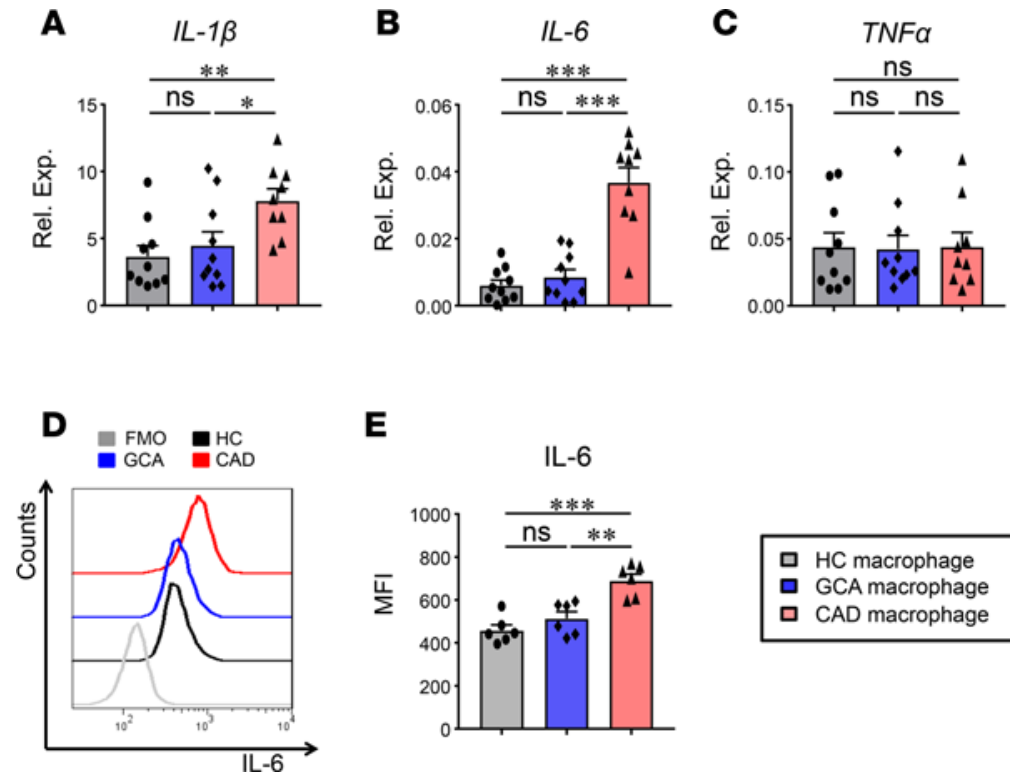

Figure 3. Monocyte-derived macrophages from CAD patients are programed to produce IL-1 $\beta$ and IL-6. Ex vivodifferentiated macrophages from HC, GCA patients, and CAD patients were stimulated with LPS/IFN- $\gamma$ for 6 hours. (A-C) Gene expression of the proinflammatory cytokines IL-1 $\beta$, IL-6, and TNF- $\alpha$ was quantified by RT-PCR. Data represent mean \pm SEM from HC and GCA ( $n=10$ each) and CAD ( $n=9$, including 5 diabetic) samples. (D and E) Flow cytometric quantification of IL-6 protein. (D) Representative histogram. (E) Summary results from 6 samples each. Three out of 6 CAD patients were diabetic. Data are mean \pm SEM and were analyzed by 1-way ANOVA with Tukey's multiple comparison test. ${ }^{*} P<0.05,{ }^{* *} P<0.01,{ }^{* *} P<0.001$. CAD, coronary artery disease; FMO, fluorescence minus one; GCA, giant cell arteritis; HC, healthy control; IFN- $\gamma$, interferon $\gamma$; IL, interleukin; LPS, lipopolysaccharide; $\mathrm{MFI}$, mean fluorescence intensity; ns, not significant; Rel. Exp., relative expression; RT-PCR, reverse transcription polymerase chain reaction; TNF- $\alpha$, tumor necrosis factor $\alpha$.

from individuals in the 3 study cohorts (Figure 4), stimulated them with IFN- $\gamma /$ LPS, and quantified the expression of PD-L1 on the cell surface. Flow cytometric analysis demonstrated PD-L1 expression on essentially the entire control macrophage population already during the resting state and robust upregulation after stimulation (Figure 4, A-C). PD-L1 surface expression was profoundly suppressed on resting and stimulated macrophages derived from GCA patients. In contrast, CAD macrophages were found to be PD-L1 high expressers during both resting and activated conditions (Figure $4, \mathrm{~A}-\mathrm{C}$ ). To test whether PD-L1 expression was also different for tissue-residing macrophages in GCA-affected arteries and in atherosclerotic plaques, we applied dual-color immunohistochemical analysis of tissue sections (Figure 4, D and E). Only a small subset of CD $68^{+}$macrophages in GCA-affected temporal arteries stained positive for PD-L1, whereas the vast majority of $\mathrm{CD} 68^{+}$cells in atherosclerotic plaque sections expressed PD-L1.

Together, these findings identified CAD macrophages as potentially immunosuppressive, whereas the $\mathrm{PD}-\mathrm{L} 1^{\text {10 }}$ status of GCA macrophages is compatible with failure of the PD-1 immune checkpoint, resulting in unopposed T cell activity.

The bioenergetics strategies of $C A D$ and GCA macrophages are divergent. In anticipation of high demands for ATP and biosynthetic precursor molecules, macrophages sensing stimulatory signals initiate a complex metabolic program. This program emphasizes glucose utilization to generate ATP and precursor compounds, needed for the synthesis of proteins, ribonucleic acids, and lipids (20). We examined how macrophages from healthy individuals and patients from the 2 disease groups adapt to M1-inducing stimuli with upregulation of the glycolytic gene module (Figure 5). Transcriptome analysis showed strong upregulation of the glycolytic machinery in CAD macrophages, whereas GCA macrophages were indistinguishable from controls (Figure 5A). Specifically, transcript concentrations for the glucose transporter GLUT1 were multifold higher in CAD macrophages compared with both GCA and control macrophages. Flow cytometric analysis confirmed strong cell surface induction of GLUT1, the dominant glucose transporter on macrophages (24) (Figure 5, B and C). There was no difference in GLUT1 expression if CAD patients were stratified for a coexistent diagnosis of diabetes mellitus (Figure 5C). The 5 key glycolytic enzymes, hexokinase 2 (HK2), phosphofructokinase 1 (PFK1), glyceraldehyde-3-phosphate dehydrogenase (GAPDH), pyruvate kinase M2 (PKM2), and lactate 
A

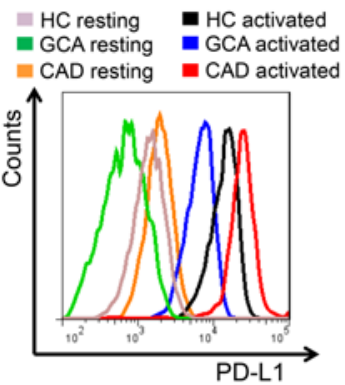

B

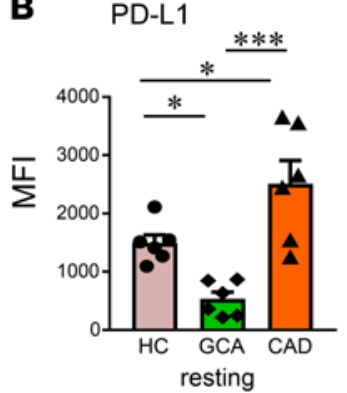

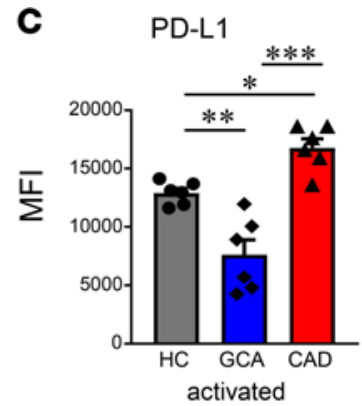

D
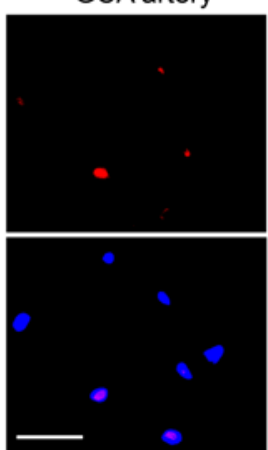

PD-L1/CD68/DAPI

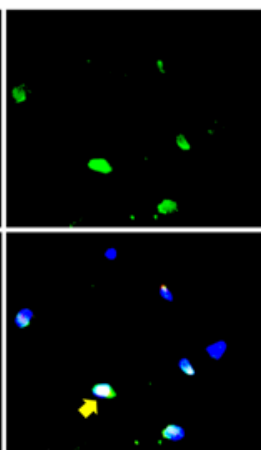

E

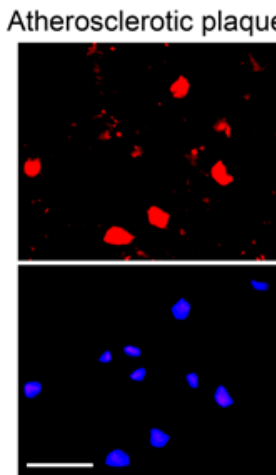

PD-L1/CD68/DAPI

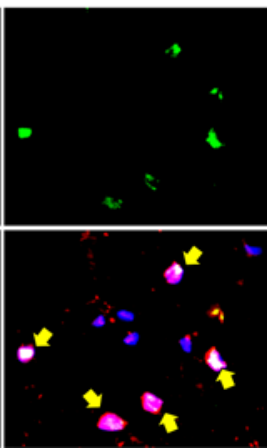

Figure 4. Differential expression of the immunoinhibitory ligand PD-L1 on monocyte-derived macrophages from GCA and CAD patients. (A-C) Monocytes from HC, patients with GCA, and patients with CAD were differentiated into macrophages. Surface PD-L1 expression was analyzed by flow cytometry on resting and activated (LPS/IFN- $\gamma, 24$ hours) macrophages. (A) Representative histograms. (B) Results from resting macrophages ( $n=6$ each). (C) Results from activated macrophages ( $n=6$ each). Three out of 6 CAD patients were diabetic. ( $\mathbf{D}$ and $\mathbf{E}$ ) Tissue sections from GCA-affected temporal arteries (D) and atherosclerotic plaques (E) were immunostained with anti-PD-L1 (red) and anti-CD68 $\mathrm{Ab}$ (green) and analyzed by fluorescence microscopy. Nuclei were labeled with DAPI. Yellow arrows indicate PD-L1-positive macrophages. Scale bars: $50 \mu \mathrm{m}$. Representative images from 4 different cases are shown. Data are mean $\pm \mathrm{SEM}$ and were analyzed by 1-way ANOVA with Tukey's multiple comparison test. ${ }^{*} P<0.05,{ }^{* *} P<0.01,{ }^{* *} P<0.001$. CAD, coronary artery disease; GCA, giant cell arteritis; HC, healthy control; IFN- $\gamma$, interferon $\gamma$; LPS, lipopolysaccharide; MFI, mean fluorescence intensity; PD-L1, programmed death ligand 1.

dehydrogenase (LDH) were all significantly more highly expressed in CAD macrophages. The 2 transcription factors c-myc and HIF-1 $\alpha$ are considered master regulators of glycolytic flux (31) and they too were highly upregulated in $\mathrm{CAD}$ macrophages (Figure 5A).

One functional consequence of enhanced glucose breakdown is the production of pyruvate, which then is imported into the mitochondria to feed the tricarboxylic acid cycle for ATP production (32). As a read-out of mitochondrial activity, we quantified production of mitochondrial ROS (Figure 5, D and E). The mean fluorescence intensity for mitoSOX was almost twice as high in CAD macrophages, consistent with intensification of mitochondrial metabolism. Accordingly, mitochondrial mass was slightly higher in CAD macrophages (Supplemental Figure 1; supplemental material available online with this article; https://doi.org/10.1172/jci.insight.123047DS1). Mitochondrial ROS production was similar in macrophages from diabetic and nondiabetic patients (Figure $5 \mathrm{E}$ ).

These data allowed the definition of a CAD-specific macrophage metabolic module characterized by high glucose absorption and high glucose utilization. GCA macrophages adopted a distinct metabolic strategy that was independent of importing and breaking down glucose.

The extracellular glucose environment determines macrophage effector functions. The close connection between the metabolic commitment to glucose import and catabolism and PD-L1 expression and IL-6 generation raised the question whether glucose availability per se directs functional differentiation of macrophages. We first asked the question whether random serum glucose levels were different in GCA and CAD patients (Figure 6A). Serum glucose was measured during the clinic visit in 33 GCA patients and $27 \mathrm{CAD}$ patients with an average of 106 and $133 \mathrm{mg} / \mathrm{dl}$, respectively. We tested whether macrophages of patients with vasculitis or $\mathrm{CAD}$ were able to make use of glucose by quantifying the cellular uptake of 


\section{A}

1) Transporte GLUT1

2) Glycolytic enzymes

HK2

PFK1

$G A P D H$

PKM2

$\mathrm{LDH}$
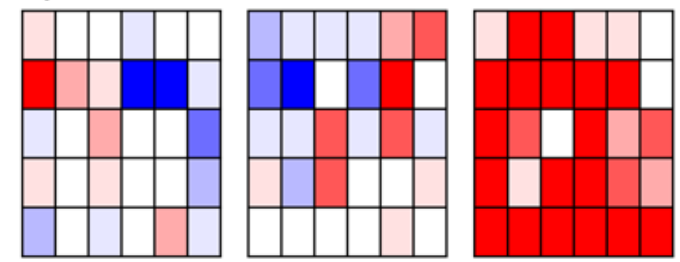

ns
ns
ns
ns
ns

$P<0.05$

$P<0.05$

$P<0.05$

$P<0.01$

$P<0.001$

3) Transcription factors

c-myc

HIF1a
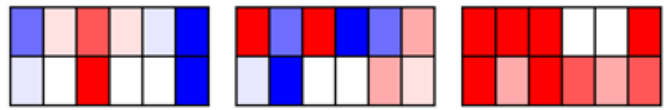

ns

ns

D

B

C
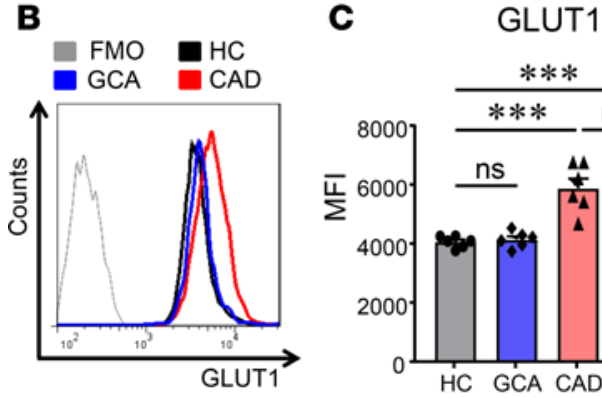

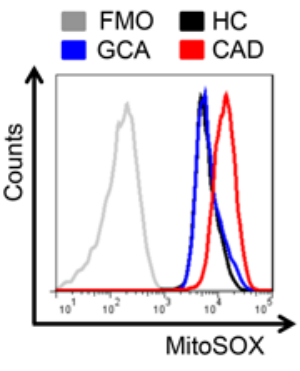

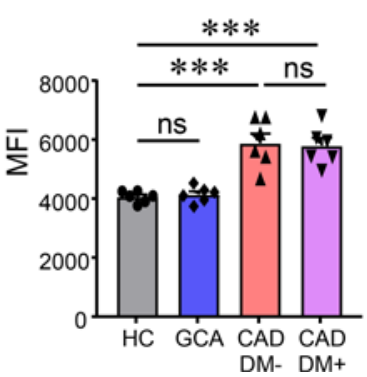

$P$ value

CAD

vs GCA

$P<0.01$

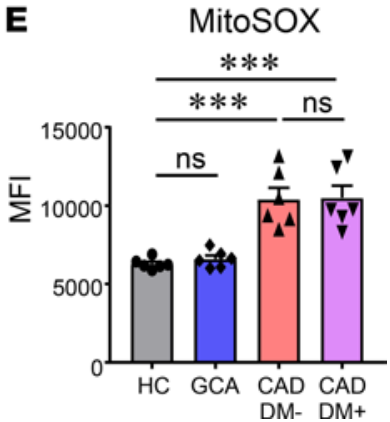

Figure 5. Monocyte-derived macrophages from CAD patients are highly efficient in glucose import and glycolytic activity. Macrophages from HC, GCA patients, and CAD patients were differentiated from blood monocytes as in Figure 1 and stimulated with LPS/IFN- $\gamma$ for 24 hours. (A) Gene transcripts for the main glucose transporter (GLUT1), the glycolytic enzymes, and transcription factors regulating glycolysis were quantified by RT-PCR ( $n=6$ each). Three out of 6 CAD patients were diabetic. Gene expression is displayed as heatmaps with data presented as $\log _{2}$ values. (B and C) Surface GLUT1 expression analyzed by flow cytometry. (B) Representative histograms. (C) Summary from 6 independent experiments. (D and E) Mitochondrial ROS production was measured by flow cytometry using mitoSOX. (D) Representative histograms. (E) Summary from 6 experiments. Data are mean \pm SEM and were analyzed by 1-way ANOVA (A) or 2-way ANOVA (C and E) with Tukey's multiple comparison test. ${ }^{* *} P<0.001$. CAD, coronary artery disease; CCL, C-C motif chemokine ligand; CXCL, C-X-C motif chemokine ligand; DM, diabetes mellitus; FMO, fluorescence minus one; HC, healthy control; HK2, hexokinase 2; CAPDH, glyceraldehyde-3-phosphate dehydrogenase; GCA, giant cell arteritis; GLUT1, glucose transporter 1; HIF-1 $\alpha$, hypoxia-inducible factor $1 \alpha$; IFN- $\gamma$, interferon $\gamma$; LDH, lactate dehydrogenase; LPS, lipopolysaccharide; MFI, mean fluorescence intensity; ns, not significant; PFK1, phosphofructokinase 1; PKM2, pyruvate kinase M2; Rel. Exp., relative expression; ROS, reactive oxygen species; RT-PCR, reverse transcription polymerase chain reaction.

the fluorescently labeled glucose analog 2-NBDG (Figure 6, B and C). 2-NBDG accumulation measured by flow cytometry was similar in control and GCA macrophages, but markedly higher in macrophages derived from CAD patients, in line with differential expression of the glucose transporter GLUT1 (Figure 5). To test whether statin medication affects glucose uptake by macrophages, we recruited a cohort of CAD patients not on statin therapy at the time of enrollment. 2-NBDG uptake was higher in CAD macrophages, unaffected by concomitant statin therapy (Figure 6C). To understand whether access to glucose is relevant for macrophage function, we quantified mitochondrial activity via mitochondrial ROS output (Figure 6D). In these experiments, glucose was titrated from 0 to $2.0 \mathrm{~g} / 1$ and D-mannitol was used as an osmotic control. Mitochondrial ROS production was directly dependent on the concentration of extracellular glucose; over the dose range of $0-2 \mathrm{~g} / 1$ glucose, mitoSOX concentrations increased by more than $50 \%$. In parallel, we determined whether accessibility to glucose influenced the production of macrophage effector molecules (Figure 6, E-G). Production of the chemokine CXCL10 appeared to be independent of the glucose concentrations in the extracellular milieu (Figure 6E). In contrast, higher glucose concentrations markedly increased the capacity of healthy and GCA-derived macrophages to synthesize IL-6 (Figure 6F) in a dose-dependent manner. A high-glucose environment increased the surface density of PD-L 1 by $42 \%$. In PD-L $1^{10}$ macrophages derived from GCA patients, even high doses of glucose could not overcome the defect (Figure 6G). To begin to understand how glucose exhibits its regulatory function, we 
A

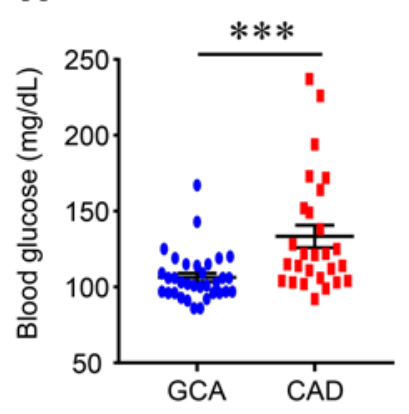

E

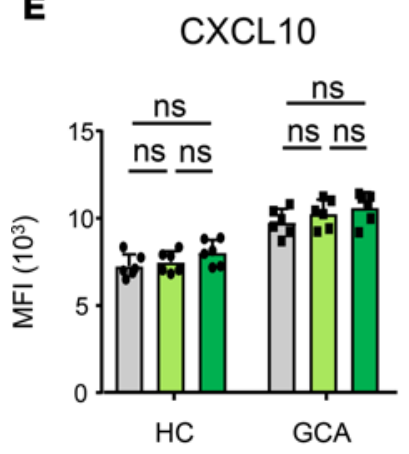

B

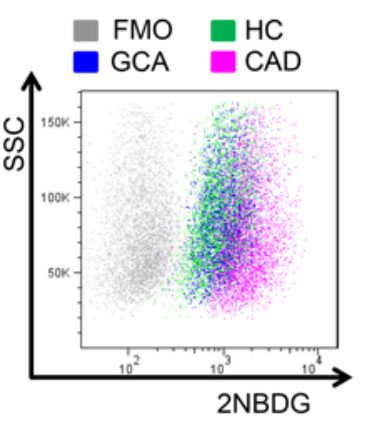

F

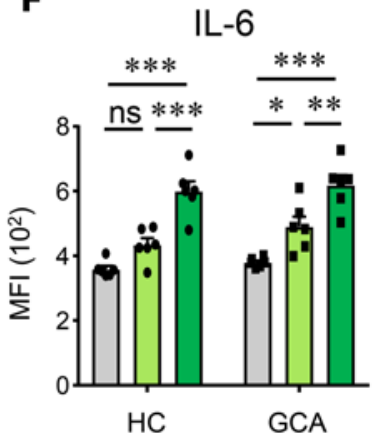

C

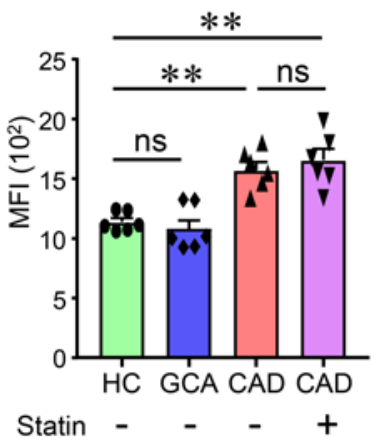

G

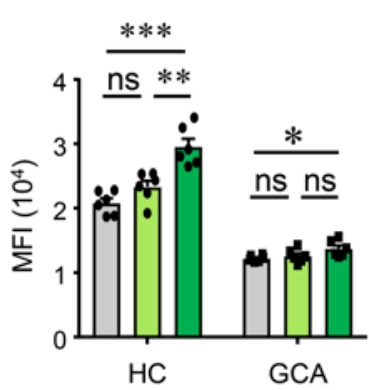

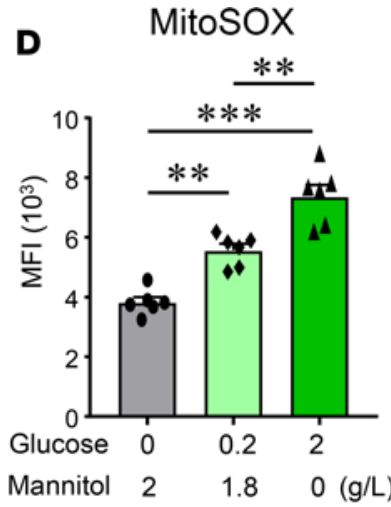

H

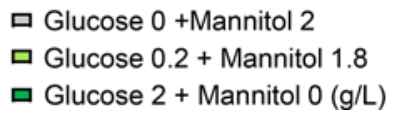

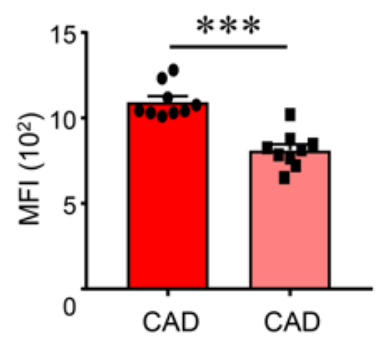

Glucose $2 \quad 0.2$

Mannitol $0 \quad 1.8(\mathrm{~g} / \mathrm{L})$

Figure 6. Glucose regulates mitochondrial activity and effector molecule expression. (A) Random serum glucose levels measured during clinic visits. Each data point represents 1 CAD or GCA patient. Twelve of 27 CAD patients had diabetes mellitus. (B and C) Ex vivo-differentiated macrophages were stimulated for 24 hours and incubated in glucose-free medium containing $5 \mu \mathrm{M}$ of the fluorescently labeled D-glucose analog 2-NBDC for 60 minutes. 2-NBDC uptake was quantified by flow cytometry. (B) Representative dot plots. (C) Summary results from 6 HC, 6 GCA patients, 6 statin-treated CAD patients, and 6 CAD patients not on a statin. (D) Ex vivo-differentiated macrophages from healthy individuals were stimulated for 6 hours in the presence of increasing concentrations of glucose. D-Mannitol was used as an osmotic control. Mitochondrial ROS production was measured by flow cytometry. Data are from 6 experiments. (E-C) Ex vivo-differentiated macrophages from HC and GCA were stimulated with LPS/IFN- $\gamma$ for 6 hours in the absence or presence of glucose. D-Mannitol was used as an osmotic control. Intracellular CXCL10 concentrations (E), intracellular IL-6 concentrations (F), and surface PD-L1 expression (G) were measured by flow cytometry. Summary from 6 independent samples. (H) Freshly isolated monocytes from CAD patients were cultured in high or low glucose concentrations for 6 hours and stimulated with LPS/IFN- $\gamma$ for 6 hours. Surface GLUT1 expression was quantified by flow cytometry. Summary from 9 (including 4 diabetic) samples. Data are mean \pm SEM and were analyzed by Mann-Whitney test (A), 2-way ANOVA with Tukey's multiple comparison test (C), 1-way ANOVA with Tukey's multiple comparison test (D-G), or paired $t$ test $(\mathbf{H}) .{ }^{*} P<0.05$; ${ }^{* *} P<0.01$; ${ }^{* * *} P<0.001$. CAD, coronary artery disease; CXCL, C-X-C motif chemokine ligand; FMO, fluorescence minus one; GCA, giant cell arteritis; GLUT1, glucose transporter 1; HC, healthy control; IFN- $\gamma$, interferon $\gamma$; IL, interleukin; LPS, lipopolysaccharide; MFI, mean fluorescence intensity; ns, not significant; PD-L1, programmed death ligand 1; ROS, reactive oxygen species; SSC, side scatter.

replaced glucose with the intermediates pyruvate, the end product of the glycolytic pathway, or succinate, an intermediate of the tricarboxylic acid cycle (Supplemental Figure 2). Pyruvate and succinate resembled glucose in leaving CXCL10 production unaffected, while boosting IL-6 production and PD-L1 expression. Consistently, pyruvate was more effective than succinate.

To further explore how glucose regulates macrophage function, we examined whether glucose with drawal results in altered cellular behavior. Ex vivo-differentiated macrophages could survive complete withdrawal from glucose for more than 6 hours (Supplemental Figure 3). The hallmark feature of CAD monocytes, the high expression of the glucose transporter GLUT1, was directly dependent on extracellular glucose (Figure 6H). Keeping GLUT1 ${ }^{\text {hi }}$ monocytes in a low-glucose environment was sufficient to downregulate GLUT1 expression.

The results identified metabolic signals as upstream regulators of the monocyte/macrophage differentiation program, indicated that monocytes and macrophages can sense glucose in their immediate surroundings, and that they can dynamically adapt to metabolic changes. 
1) Chemokines

CXCL9

CXCL10

CXCL11

2) Cytokines

$I L-1 \beta$
$I L-6$
$T N F \alpha$
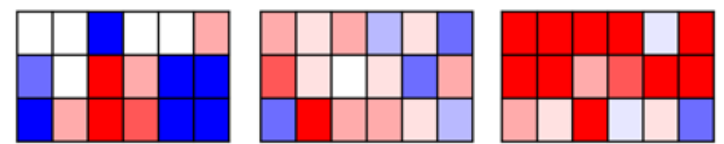

3) Glycolysis

GLUT1
HK2
PKM2
LDH
$c-m y c$
HIF1a
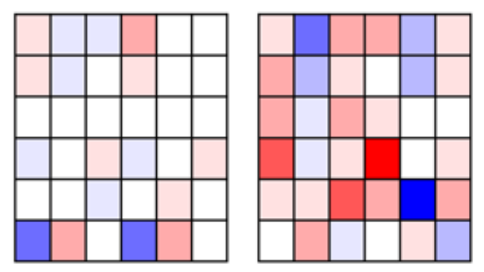

$\log _{2}$

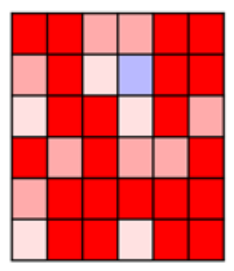

ns

ns

ns

ns

ns

ns ns

ns

ns

$P$ value $P$ value $P$ value

GCA CAD CAD

vs $\mathrm{HC}$ vs $\mathrm{HC}$ vs GCA

$P<0.01 \quad P<0.001 \quad$ ns

$P<0.05 \quad P<0.001 \quad n s$

$\mathrm{P}<0.05 \quad \mathrm{P}<0.05 \quad$ ns

$\begin{array}{ccc}\text { ns } & \mathrm{P}<0.05 & \mathrm{P}<0.05 \\ \text { ns } & \mathrm{P}<0.05 & \mathrm{~ns} \\ \text { ns } & \text { ns } & \mathrm{ns} \\ & & \\ \text { ns } & \mathrm{P}<0.01 & \mathrm{P}<0.01 \\ \text { ns } & \mathrm{P}<0.01 & \mathrm{P}<0.01 \\ \text { ns } & \mathrm{P}<0.01 & \mathrm{P}<0.05 \\ \text { ns } & \mathrm{P}<0.01 & \mathrm{nS} \\ \text { ns } & \mathrm{P}<0.01 & \mathrm{P}<0.01 \\ \text { ns } & \mathrm{P}<0.01 & \mathrm{P}<0.05\end{array}$

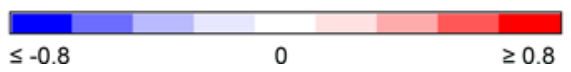

Figure 7. Gene expression profiles are preserved from monocytes to macrophages. Blood $\mathrm{CD} 14^{+}$monocytes were freshly isolated from the 3 study cohorts ( $n=6$ each) and stimulated with LPS/IFN- $\gamma$. Three out of 6 CAD patients had diabetes mellitus. Transcripts of chemokines (CXCL9, CXCL10, and CXCL11), cytokines (IL-1 $1 \beta$, IL-6, and TNF- $\alpha$ ) and glycolysis-associated genes (CLUT1, HK2, PKM2, LDH, c-myc, and HIF-1 $\alpha$ ) were determined by RT-PCR. Heatmap display with gene expression data presented as $\log _{2}$ values. Data were analyzed by 1-way ANOVA with Tukey's multiple comparison test. $C A D$, coronary artery disease; $C X C L, C-X-C$ motif chemokine ligand; $H C$, healthy control; HK2: hexokinase 2; GAPDH, glyceraldehyde-3-phosphate dehydrogenase; GCA, giant cell arteritis; GLUT1, glucose transporter 1; HIF-1 $\alpha$, hypoxia-inducible factor $1 \alpha$; IFN- $\gamma$, interferon $\gamma$; IL, interleukin; LDH, lactate dehydrogenase; LPS, lipopolysaccharide; ns, not significant; PFK1, phosphofructokinase 1; PKM2, pyruvate kinase M2; RT-PCR, reverse transcription polymerase chain reaction; TNF- $\alpha$, tumor necrosis factor $\alpha$.

Functional commitments and metabolic programming are preserved from monocytes to macrophages. To investigate whether the differences in functional commitment and metabolic fitness between CAD and GCA macrophages could possibly be the result of the in vitro differentiation and at which point in their life cycle macrophages diverge towards a defined functional profile, we examined freshly isolated blood monocytes (Figure 7). Monocytes were purified immediately after harvesting of the blood samples and subjected to transcriptome analysis. Monocytes from CAD and GCA patients expressed a distinct pattern of CXCL9, CXCL10, and CXCL11 upregulation compared with healthy monocytes, recapitulating profiles established for differentiated macrophages (Figure 1). Monocytes derived from CAD patients, but not those from GCA patients, were primed to produce increased levels of IL-1 $\beta$ and IL- 6 transcripts, again resembling differentiated macrophages (Figure 7). Similarly, monocytes collected from CAD patients had upregulated the glycolytic machinery and had acquired a metabolic signature identical to the differentiated macrophages (Figure 7), separating them from healthy control and GCA monocytes.

These data identified the bone marrow, the birthplace of monocytes, as a fate-determining microenvironment and suggested that the disease-specific signature is imprinted early in the life cycle of macrophages.

\section{Discussion}

Infiltration of macrophages into the vessel wall is a hallmark of vascular inflammation, wherein these myeloid cells are recognized as key drivers of disease. Macrophages have broad sensing capabilities and participate in multiple disease pathways. Here, we investigated 2 classical vascular disorders associated with the presence of macrophages in vessel wall infiltrates: large-vessel vasculitis and atherosclerosis. Both conditions are recognized as inflammatory entities, with CRP measurement used widely to estimate disease burden $(27,33)$. Large-vessel vasculitis is an aggressive inflammatory condition characterized by its rapidly progressive clinical course and the speed of vessel wall remodeling, whereas atherosclerosis begins 
in young adults and slowly progresses over decades with a smoldering course. A careful comparison of monocytes, macrophages, and tissue-residing macrophages revealed a disease-specific patterning of core inflammatory functions, such as production of chemokines, cytokines, and expression of the immunomodulatory ligand PD-L1. Unexpectedly, monocytes were already imprinted with the disease-specific signature and memorized their functional commitment as they differentiated into macrophages. We identified glucose availability and uptake as a critical determinant in directing macrophage differentiation, placing metabolic signals upstream of immunoinflammatory effector functions. Glucose-dependent conditioning of monocytes must occur outside of the vessel wall lesions, redirecting attention to the bone marrow and the circulation, rather than the inflamed tissue site.

Monocytes and macrophages from both patient cohorts were prone to producing chemokines, especially $\mathrm{T}$ cell-attracting chemokines involved in the assembly of chronic inflammatory tissue lesions. In vasculitic arteries and in atherosclerotic plaques, essentially every CD68 ${ }^{+}$cell was able to secrete CXCL10, a chemokine known to recruit $\mathrm{T}$ cells committed to the Th1 lineage. Like differentiated macrophages, freshly isolated monocytes were already committed to produce CXCL9, CXCL10, and CXCL11. Monocytes reside in the bone marrow for a brief period before being released into the circulation (34). CXCL10-producing monocytes would be able to assemble cellular infiltrates of CXCL10-responsive populations in the bone marrow. Abnormalities in bone marrow stromal cells have been described in GCA patients (35). Also, neutrophils in GCA patients display an inflammatory phenotype (36), compatible with involvement of the bone marrow microenvironment in this vasculitis. The possibility of a bone marrow defect associated with the risk for atherosclerotic cardiovascular disease has been raised by recent studies reporting enrichment of clonal hematopoiesis of indeterminate potential (CHIP) carriers among CAD patients (37), emphasizing the potential mechanistic connection between clonal hematopoiesis and cardiovascular disease. Similarly, ${ }^{18}$ F-FDG PET imaging has indicated that in patients with $\mathrm{CAD}$, bone marrow uptake of the tracer is enhanced (38), consistent with low-grade inflammation in the bone marrow space. Additional studies are needed to understand how the bone marrow contributes to vascular disease. In experiments aimed at modifying extracellular glucose levels, CXCL10 expression was unaffected by rising glucose availability, in line with the finding that CAD and GCA patients shared induction of CXCL10 but differed fundamentally in enhancement of the glycolytic machinery. These data suggest that signals different from glucose may modulate monocyte function in the bone marrow environment, priming them to specialize in chemokine production.

Cytokines are principle mediators of tissue inflammation, to the extent that they are useful therapeutic targets for antiinflammatory therapy. Confirming current paradigms of the critical role of both IL- $1 \beta$ and IL-6 in pathogenic events of CAD (39), monocytes and macrophages derived from CAD patients surpassed healthy monocytes and macrophages in the production of the 2 proinflammatory cytokines. Blocking IL-1 $\beta$ protects at-risk patients from recurrent cardiovascular complications (29). Surprisingly, monocytes and macrophages from GCA patients were similar to healthy cells. Elevated IL- 6 concentrations have been considered a robust marker of vasculitic activity and IL- 6 receptors are blocked therapeutically in the attempt to spare glucocorticoids (40). These data suggest that IL-6 in GCA patients may derive from other cell populations. Myriad cells can produce IL-6 and respond to IL-6 (41) and proinflammatory as well as regenerative and protective responses have been assigned to the cytokine (42). The findings confirm prior studies that failed to find evidence for IL-6-dependent signaling in inflamed arteries (9), supporting the concept that IL-6 may not be a critical disease driver in the vascular lesions of large-vessel vasculitis.

A notable difference between monocytes and macrophages from CAD and GCA patients was the divergent expression of PD-L1, an immunoinhibitory ligand. Abundance of PD-L1 in CAD macrophages and lack of it in GCA macrophages was maintained in the tissue-residing cells. Interestingly, glucose concentration was critical in enhancing PD-L1 expression in CAD and healthy macrophages but could not overcome the PD-L1 deficiency typical for GCA macrophages. Besides macrophages, dendritic cells of GCA patients are $\mathrm{PD}-\mathrm{L} 1^{\text {lo }}(15)$, suggesting an abnormality in the regulation of the PD-L1-encoding gene in this disease. The prediction is that macrophages in CAD patients combine proinflammatory and immunosuppressive functions, which should result in exuberant innate immunity and deficient adaptive immunity. Evidence suggests that the macrophages of CAD patients are indeed inefficient in supporting antiviral $\mathrm{T}$ cell immunity (25). The risk for uncontrolled inflammation in GCA patients should cause a different pattern of disease: exuberant innate immunity combined with unopposed T cell immunity. Given the importance of the PD-1 pathway in T cell-mediated antitumor responses $(30,43)$, the 2 patient populations should differ in their susceptibility to developing malignancies, a prediction supported by currently available epidemiological data. 
Specifically, a diagnosis of GCA is not associated with an increased risk for having cancer (44-46), whereas coronary heart disease shares common genetic risk factors with cancer susceptibility $(47,48)$.

Glucose has emerged as a major upstream regulator of disease-specific signatures in CAD and GCA. There is overwhelming evidence that hyperglycemia is a strong risk factor for $\operatorname{CAD}(49,50)$, whereas prospective cohort studies in Sweden have associated low body mass index, hypoglycemia, and hypolipidemia with later-life diagnosis of GCA (51). These data are in line with the current observation that extracellular glucose concentrations can deviate macrophage differentiation towards high production of IL-1 $\beta$, IL-6, and high expression of PD-L1. In preclinical models, evidence has been provided that hyperglycemia can drive enhanced production and activation of myelopoiesis by regulating proliferative activity of bone marrow precursor cells $(52,53)$, in line with data presented here that monocytes in CAD patients already are imprinted with a proinflammatory program. Experiments that measured the impact of changing glucose concentrations in the extracellular fluid indicated that human monocytes and macrophages can sense glucose availability and dynamically adapt their glycolytic machinery. A key parameter seems to be the surface density of GLUT1, the dominant glucose transporter in human macrophages (24). Even under physiologic conditions, monocytes and macrophages have spare capacity to respond to rising glucose concentrations. Under chronic overfeeding conditions (e.g., hyperglycemia in CAD patients), monocytes and macrophages upregulate glucose uptake and utilization. Glucose-enriched microenvironments appear to drive monocytes and macrophages towards chemokine and cytokine production, while impairing $\mathrm{T}$ cell immunity by bolstering surface PD-L1. Experiments that demonstrated that glucose withdrawal was sensed and triggered reduced glucose import encourage considerations to use manipulation of glucose in the extracellular milieu as a strategy to balance pro- and antiinflammatory signals. Plasticity of monocytes and macrophages in responding to fate-determining metabolic surroundings opens the possibility for therapeutic intervention, using metabolic interference to direct the immune system towards or away from inflammatory immunity.

\section{Methods}

Patients and controls. The study cohort included 42 patients with GCA and 43 patients with CAD. Patients with GCA were diagnosed based on temporal artery biopsy with typical histopathological findings (15). Patients with CAD had at least 1 documented myocardial infarction and were enrolled more than 90 days after an ischemic event $(24,25)$. Demographic characteristics of GCA and CAD patients are summarized in Table 1. Healthy individuals aged 60 years and older were recruited from the Preventive Medicine Program and the Blood and Platelet Donor program of the Stanford Blood Center $(n=53)$. They had no personal or familial history of diabetes, autoimmune disease, cancer, chronic viral infection, or any other chronic inflammatory disorder.

Cell culture. Peripheral blood mononuclear cells (PBMCs) were isolated and macrophages were differentiated from $\mathrm{CD}_{14}{ }^{+}$monocytes as previously described (24, 25). Macrophages were stimulated with $100 \mathrm{U} /$ $\mathrm{ml}$ IFN- $\gamma$ (catalog 11725-HNAS, Sino Biologicals) and $100 \mathrm{ng} / \mathrm{ml}$ LPS (catalog L2630-10MG, Sigma-Aldrich) for the indicated time periods.

Quantitative RT-PCR. Methods for RT-PCR have been previously described $(24,25)$. Gene transcript numbers were adjusted relative to $\beta$-actin transcripts. The primers used in this study are listed in Supplemental Table 1.

Flow cytometry. Expression of surface molecules and intracellular proteins was measured using a BD LSRFortessa, and data were analyzed with FlowJo (Tree Star). To evaluate intracellular protein, the cells were fixed and permeabilized with the BD Cytofix/Cytoperm Kit. PE-anti-IP-10 (CXCL10) antibody (catalog 555049) and Alexa Fluor 647-anti-GLUT1 antibody (catalog 566580) were obtained from BD Pharmingen, and FITC-anti-IL-6 antibody (catalog 501104) and APC-anti-CD274 (PD-L1) antibody (catalog 329708) were from BioLegend.

Glucose and metabolic intermediates. D-Mannitol (catalog M4125-100G, Sigma-Aldrich) was used for osmotic control of glucose (catalog A24940-01, Gibco/Thermo Fisher Scientific). Sodium pyruvate was purchased from Santa Cruz Biotechnology (catalog sc-208397A) and succinic acid (catalog S3674-100G) was from Sigma-Aldrich.

Measurement of mitochondrial mass and mitochondrial ROS. Macrophages were stimulated with IFN- $\gamma /$ LPS for 24 hours, and then stained with 500 nM MitoTracker Green FM (catalog M7514, Molecular Probes/Thermo Fisher Scientific) for 15 minutes or $5 \mu \mathrm{M}$ MitoSOX Red (catalog M36008, Molecular Probes/Thermo Fisher Scientific) for 10 minutes, washed with PBS, and analyzed by flow cytometry. 
Table 1. Clinical characteristics of patients enrolled in this study

\begin{tabular}{|c|c|c|}
\hline & Giant cell arteritis (GCA) & Coronary artery disease (CAD) \\
\hline Parameters ${ }^{A}$ & $n=42$ & $n=43$ \\
\hline Age (mean \pm SD) & $74.5 \pm 8.9$ & $67.3 \pm 9.8$ \\
\hline Male/Female & $14: 28$ & $38: 5$ \\
\hline \multicolumn{3}{|l|}{ Ethnicity } \\
\hline White & $38(90.5 \%)$ & $37(86.0 \%)$ \\
\hline Hispanic & $3(7.1 \%)$ & $2(4.7 \%)$ \\
\hline Asian & $1(2.4 \%)$ & $3(7.0 \%)$ \\
\hline BMI (mean \pm SD) & $26.3 \pm 3.4$ & $30.7 \pm 7.0$ \\
\hline Diabetes mellitus & $1(2.4 \%)$ & $21(48.8 \%)$ \\
\hline Hypertension & $7(16.7 \%)$ & $33(76.7 \%)$ \\
\hline Hyperlipidemia & $14(33.3 \%)$ & $38(88.4 \%)$ \\
\hline \multicolumn{3}{|l|}{ GCA-related } \\
\hline Disease duration (month, mean \pm SD) & $5.83 \pm 4.32$ & \\
\hline ESR (mean $\pm \mathrm{SD}, \mathrm{mm} / \mathrm{h})$ & $43.43 \pm 24.24$ & \\
\hline CRP (mean \pm SD, mg/dl) & $4.66 \pm 4.30$ & \\
\hline Headache & $21(50.0 \%)$ & \\
\hline Eye involvement & $15(35.7 \%)$ & \\
\hline Aortic/large vessel involvement & $26(61.9 \%)$ & \\
\hline Polymyalgia rheumatica & $34(81.0 \%)$ & \\
\hline Untreated & $14(33.3 \%)$ & \\
\hline Prednisone (mg/day, mean \pm SD) & $5.32 \pm 6.15$ & \\
\hline Second immunosuppressant & $11(26.2 \%)$ & \\
\hline \multicolumn{3}{|l|}{ CAD-related } \\
\hline Family history of CAD & & $20(46.5 \%)$ \\
\hline \multicolumn{3}{|l|}{ Smoking } \\
\hline Current & & $3(7.0 \%)$ \\
\hline Former & & $28(65.1 \%)$ \\
\hline \multicolumn{3}{|l|}{ Treatment } \\
\hline ACE inhibitor & & $14(32.6 \%)$ \\
\hline ATII receptor blocker & & $10(23.3 \%)$ \\
\hline$\beta$-Blocker & & $27(62.8 \%)$ \\
\hline Ca-antagonist & & $7(16.3 \%)$ \\
\hline Diuretic & & $10(18.6 \%)$ \\
\hline Anti-platelet & & $34(79.0 \%)$ \\
\hline Statin & & $32(74.4 \%)$ \\
\hline Sulfonylurea & & $2(4.7 \%)$ \\
\hline Insulin & & $3(7.0 \%)$ \\
\hline
\end{tabular}

Measurement of glucose uptake. Macrophages were stimulated with IFN- $\gamma /$ LPS for 24 hours and incubated with glucose-free RPMI medium containing $5 \mu \mathrm{M}$ fluorescent D-glucose analog 2-NBDG (2-[N-(7-nitrobenz2-oxa-1,3-diazol-4-yl) amino]-2-deoxy D-glucose) (catalog 11046, Cayman Chemical) for 60 minutes. Macrophages were washed with PBS and fluorescence intensities were analyzed by flow cytometry.

Apoptosis assay. Macrophages were stimulated with IFN- $\gamma /$ LPS for 6 hours in the absence or presence of glucose ( $2 \mathrm{~g} / \mathrm{l})$, suspended in binding buffer (BD), and stained with 7-AAD (BD) and Pacific Blueanti-annexin V (BioLegend). Fluorescence intensities were analyzed by flow cytometry.

Immunohistochemistry. Immunohistochemistry was performed as previously published (25). Formalin-fixed, paraffin-embedded tissue sections of biopsy-positive temporal artery or aortic atherosclerotic plaque were deparaffinized with xylene and ethanol, and antigen retrieval was performed with Target Retrieval Solution (catalog S1699, Dako/Agilent). After blocking with 5\% normal goat serum (catalog 31873, Invitrogen/Thermo Fisher Scientific), sections were incubated with unconjugated primary antibody overnight. Primary antibody binding was visualized by staining with Alexa Fluor 488-conjugated goat 
anti-rabbit IgG (catalog A11008, Life Technologies/Thermo Fisher Scientific) or Alexa Fluor 546 goat antimouse IgG (catalog A11003, Life Technologies/Thermo Fisher Scientific) secondary antibodies for 2 hours, and mounted with DAPI-containing mounting medium (catalog P36961, Molecular Probes/Thermo Fisher Scientific). Tissue sections were examined using confocal microscopy (LSM 710, Carl Zeiss) or fluorescence microscopy (Olympus). Antibodies used in this study are listed in Supplemental Table 2.

Statistics. All data were evaluated for normality by D'Agostino-Pearson omnibus normality testing. Based on the normality, statistical significance was calculated by unpaired or paired 2-tailed Student's $t$ test or the Mann-Whitney test as appropriate. When we compared multiple groups, 1-way or 2-way ANOVA with Tukey's multiple comparison test was employed as appropriate. All analyses were performed using Prism software (GraphPad Software version 7). $P$ values less than 0.05 were considered significant.

Study approval. The study was approved by the Institutional Review Board at Stanford University and written informed consent was obtained from all participants.

\section{Author contributions}

RW, $\mathrm{MH}, \mathrm{HZ}$, and MZ performed experiments, and RW was responsible for data analysis. BBW and JCG enrolled patients and GJB provided expertise in case definition and tissue collection. CMW, JJG, and DGH conceived the study, designed experiments, and analyzed data. CMW, RW, and JJG wrote the manuscript.

Address correspondence to: Cornelia M. Weyand, Department of Medicine, Stanford University School of Medicine, CCSR Building Room 2225, 269 Campus Drive West, Stanford, California 94305-5166, USA. Phone: 650.723.9027; Email: cweyand@stanford.edu.

1. Gordon S, Taylor PR. Monocyte and macrophage heterogeneity. Nat Rev Immunol. 2005;5(12):953-964.

2. Laskin DL, Sunil VR, Gardner CR, Laskin JD. Macrophages and tissue injury: agents of defense or destruction? Annu Rev Pharmacol Toxicol. 2011;51:267-288.

3. Wynn TA, Chawla A, Pollard JW. Macrophage biology in development, homeostasis and disease. Nature. 2013;496(7446):445-455.

4. Verreck FA, et al. Human IL-23-producing type 1 macrophages promote but IL-10-producing type 2 macrophages subvert immunity to (myco)bacteria. Proc Natl Acad Sci USA. 2004;101(13):4560-4565.

5. Mantovani A, Sica A, Sozzani S, Allavena P, Vecchi A, Locati M. The chemokine system in diverse forms of macrophage activation and polarization. Trends Immunol. 2004;25(12):677-686.

6. Lee CG, et al. Interleukin-13 induces tissue fibrosis by selectively stimulating and activating transforming growth factor beta(1). J Exp Med. 2001;194(6):809-821.

7. Buttgereit F, Dejaco C, Matteson EL, Dasgupta B. Polymyalgia rheumatica and giant cell arteritis: A systematic review. JAMA. 2016;315(22):2442-2458.

8. Weyand CM, Goronzy JJ. Clinical practice. Giant-cell arteritis and polymyalgia rheumatica. NEngl J Med. 2014;371(1):50-57.

9. Zhang H, Watanabe R, Berry GJ, Tian L, Goronzy JJ, Weyand CM. Inhibition of JAK-STAT signaling suppresses pathogenic immune responses in medium and large vessel vasculitis. Circulation. 2018;137(18):1934-1948.

10. Ma-Krupa W, Jeon MS, Spoerl S, Tedder TF, Goronzy JJ, Weyand CM. Activation of arterial wall dendritic cells and breakdown of self-tolerance in giant cell arteritis. J Exp Med. 2004;199(2):173-183.

11. Weyand CM, Goronzy JJ. Immune mechanisms in medium and large-vessel vasculitis. Nat Rev Rheumatol. 2013;9(12):731-740.

12. Ciccia F, et al. Difference in the expression of IL-9 and IL-17 correlates with different histological pattern of vascular wall injury in giant cell arteritis. Rheumatology (Oxford). 2015;54(9):1596-1604.

13. Deng J, Younge BR, Olshen RA, Goronzy JJ, Weyand CM. Th17 and Th1 T-cell responses in giant cell arteritis. Circulation. 2010;121(7):906-915.

14. Terrier B, et al. Interleukin-21 modulates Th1 and Th17 responses in giant cell arteritis. Arthritis Rheum. 2012;64(6):2001-2011.

15. Zhang H, et al. Immunoinhibitory checkpoint deficiency in medium and large vessel vasculitis. Proc Natl Acad Sci USA. 2017;114(6):E970-E979.

16. Corbera-Bellalta M, et al. Blocking interferon $\gamma$ reduces expression of chemokines CXCL9, CXCL10 and CXCL11 and decreases macrophage infiltration in ex vivo cultured arteries from patients with giant cell arteritis. Ann Rheum Dis. 2016;75(6):1177-1186.

17. Rittner HL, Hafner V, Klimiuk PA, Szweda LI, Goronzy JJ, Weyand CM. Aldose reductase functions as a detoxification system for lipid peroxidation products in vasculitis. J Clin Invest. 1999;103(7):1007-1013.

18. Watanabe R, et al. Matrix metalloprotease-9 (MMP-9)-producing monocytes enable T cells to invade the vessel wall and cause vasculitis. Circ Res. 2018;123:700-715.

19. Libby P, Ridker PM, Hansson GK. Progress and challenges in translating the biology of atherosclerosis. Nature. 2011;473(7347):317-325.

20. Koelwyn GJ, Corr EM, Erbay E, Moore KJ. Regulation of macrophage immunometabolism in atherosclerosis. Nat Immunol. 2018;19(6):526-537.

21. Colin S, Chinetti-Gbaguidi G, Staels B. Macrophage phenotypes in atherosclerosis. Immunol Rev. 2014;262(1):153-166.

22. Tabas I, Lichtman AH. Monocyte-macrophages and T cells in atherosclerosis. Immunity. 2017;47(4):621-634.

23. Hansson GK. Inflammation, atherosclerosis, and coronary artery disease. N Engl J Med. 2005;352(16):1685-1695

24. Shirai T, et al. The glycolytic enzyme PKM2 bridges metabolic and inflammatory dysfunction in coronary artery disease. $J$ Exp 
Med. 2016;213(3):337-354.

25. Watanabe R, et al. Pyruvate controls the checkpoint inhibitor PD-L1 and suppresses T cell immunity. J Clin Invest. 2017;127(7):2725-2738

26. Charo IF, Taubman MB. Chemokines in the pathogenesis of vascular disease. Circ Res. 2004;95(9):858-866

27. Ridker PM. Inflammation, C-reactive protein, and cardiovascular disease: moving past the marker versus mediator debate. Circ Res. 2014;114(4):594-595.

28. Roche NE, Fulbright JW, Wagner AD, Hunder GG, Goronzy JJ, Weyand CM. Correlation of interleukin-6 production and disease activity in polymyalgia rheumatica and giant cell arteritis. Arthritis Rheum. 1993;36(9):1286-1294.

29. Ridker PM, et al. Antiinflammatory therapy with canakinumab for atherosclerotic disease. N Engl J Med. 2017;377(12):1119-1131.

30. Baumeister SH, Freeman GJ, Dranoff G, Sharpe AH. Coinhibitory pathways in immunotherapy for cancer. Annu Rev Immunol. 2016;34:539-573.

31. DeBerardinis RJ, Lum JJ, Hatzivassiliou G, Thompson CB. The biology of cancer: metabolic reprogramming fuels cell growth and proliferation. Cell Metab. 2008;7(1):11-20.

32. Martinez-Outschoorn UE, Peiris-Pagés M, Pestell RG, Sotgia F, Lisanti MP. Cancer metabolism: a therapeutic perspective. Nat Rev Clin Oncol. 2017;14(1):11-31.

33. Schmidt J, Warrington KJ. Polymyalgia rheumatica and giant cell arteritis in older patients: diagnosis and pharmacological management. Drugs Aging. 2011;28(8):651-666.

34. Patel AA, et al. The fate and lifespan of human monocyte subsets in steady state and systemic inflammation. J Exp Med. 2017;214(7):1913-1923.

35. Orphanos V, Andonopoulos AP, Kourakli A, Zoumbos NC. Cytokine gene expression in the marrow stromal cells of patients with giant cell arteritis. Clin Exp Rheumatol. 1998;16(6):683-688.

36. Nadkarni S, Dalli J, Hollywood J, Mason JC, Dasgupta B, Perretti M. Investigational analysis reveals a potential role for neutrophils in giant-cell arteritis disease progression. Circ Res. 2014;114(2):242-248.

37. Jaiswal S, et al. Clonal hematopoiesis and risk of atherosclerotic cardiovascular disease. N Engl J Med. 2017;377(2):111-121.

38. van der Valk FM, et al. Increased haematopoietic activity in patients with atherosclerosis. Eur Heart J. 2017;38(6):425-432.

39. Ridker PM. From C-reactive protein to interleukin-6 to interleukin-1: Moving upstream to identify novel targets for atheroprotection. Circ Res. 2016;118(1):145-156.

40. Stone JH, et al. Trial of tocilizumab in giant-cell arteritis. N Engl J Med. 2017;377(4):317-328.

41. Kishimoto T. Interleukin-6: from basic science to medicine--40 years in immunology. Annu Rev Immunol. 2005;23:1-21.

42. Scheller J, Chalaris A, Schmidt-Arras D, Rose-John S. The pro- and anti-inflammatory properties of the cytokine interleukin-6. Biochim Biophys Acta. 2011;1813(5):878-888.

43. Pardoll DM. The blockade of immune checkpoints in cancer immunotherapy. Nat Rev Cancer. 2012;12(4):252-264.

44. Kermani TA, et al. Cancer preceding giant cell arteritis: a case-control study. Arthritis Rheum. 2010;62(6):1763-1769.

45. Lee YH, Song GG. Overall and cause-specific mortality in giant cell arteritis: A meta-analysis [published online ahead of print March 20, 2018]. Z Rheumatol. https://doi.org/10.1007/s00393-018-0440-7.

46. Li L, Neogi T, Jick S. Giant cell arteritis and vascular disease-risk factors and outcomes: a cohort study using UK Clinical Practice Research Datalink. Rheumatology (Oxford). 2017;56(5):753-762.

47. Koene RJ, Prizment AE, Blaes A, Konety SH. Shared risk factors in cardiovascular disease and cancer. Circulation. 2016;133(11):1104-1114.

48. Masoudkabir F, et al. Cardiovascular disease and cancer: Evidence for shared disease pathways and pharmacologic prevention. Atherosclerosis. 2017;263:343-351.

49. Beckman JA, Creager MA, Libby P. Diabetes and atherosclerosis: epidemiology, pathophysiology, and management. JAMA. 2002;287(19):2570-2581.

50. Shechter M, Merz CN, Paul-Labrador MJ, Kaul S. Blood glucose and platelet-dependent thrombosis in patients with coronary artery disease. J Am Coll Cardiol. 2000;35(2):300-307.

51. Jakobsson $\mathrm{K}$, et al. Body mass index and the risk of giant cell arteritis: results from a prospective study. Rheumatology (Oxford). 2015;54(3):433-440.

52. Barrett TJ, Murphy AJ, Goldberg IJ, Fisher EA. Diabetes-mediated myelopoiesis and the relationship to cardiovascular risk. Ann N Y Acad Sci. 2017;1402(1):31-42.

53. Nagareddy PR, et al. Hyperglycemia promotes myelopoiesis and impairs the resolution of atherosclerosis. Cell Metab. 2013;17(5):695-708. 